\title{
Relacionando Modelagem de Tópicos e Classificação de Sentimentos para Análise de Mensagens do Twitter Durante a Pandemia da COVID-19
}

\author{
Matheus Adler Soares Pinto \\ matheusadler@gmail.com \\ Universidade Estadual do Maranhão \\ Antonio José G. Busson \\ busson@telemidia.puc-rio.br \\ TeleMidia/PUC-Rio
}

\begin{abstract}
In 2020, COVID-19 pandemic is one of the most talked-about subjects on social networks. This subject has generated discussions of great importance about politics, economics, medical advances, people's awareness, preventive techniques, etc. Using sentiment analysis and topic modeling techniques, in this paper, we aim to present an analysis of the messages from the social network Twitter during the pandemic of COVID-19. For this, we use a tweets dataset to train a sentiment classifier and then use the NMF algorithm to perform the interest topic generation.
\end{abstract}

\section{KEYWORDS}

sentiment analysis, covid-19, coronavírus, tweets

\section{INTRODUÇÃO}

A COVID-19 é a doença causada pelo novo vírus da classificação SARS-COV2. Conceituada como uma síndrome respiratória aguda grave potencialmente fatal, surgiu em Wuhan, na China, em dezembro de 2019, e desde então vem se espalhando por todos os continentes do mundo [9], tornando-se um dos assuntos mais comentados do ano de 2020. Com isso, boa parte das discussões e ações da sociedade têm girado em torno desse tema. Merecem citação as medidas governamentais para enfrentar o período pandêmico; os hábitos adquiridos em todas as regiões ao redor do mundo; o quadro de informações diárias; o andamento das pesquisas para o tratamento da doença; as técnicas de prevenção; etc. Acompanha esse cenário a grande quantidade de informações que circula diariamente nas redes sociais. A compreenssão sobre a opinião dos usuários dessas redes é um trabalho desafiador, mas que pode trazer diversos benefícios no sentido de aprimorar o entendimento do impacto que a pandemia tem causado nas populações de todo planeta.

O twitter é uma das principais redes sociais do mundo, contando com cerca de 316 milhões de usuários ativos. A rede permite compartilhar diversas formas de conteúdo, seja por meio dos tweets, que são publicações de mensagens textuais, ou dos retweets, onde um usuário repercute a publicação de outros usuários. Por conta disso, o twitter se torna uma fonte rica para o entendimento dos

In: XVII Workshop de Trabalhos de Iniciação Científica (WTIC 2020), São Luís, Brasil Anais Estendidos do Simpósio Brasileiro de Sistemas Multimídia e Web (WebMedia)

Porto Alegre: Sociedade Brasileira de Computação, 2020.

(c) 2020 SBC - Sociedade Brasileira de Computação.

ISSN 2596-1683

\author{
Antonio Fernando Lavareda Jacob Junior \\ antoniojunior@professor.uema.br \\ Universidade Estadual do Maranhão \\ Sérgio Colcher \\ colcher@inf.puc-rio.br \\ TeleMidia/PUC-Rio
}

sentimentos dos usuários e, em especial, para o conhecimento dos principais tópicos relacionados ao tema da pandemia que têm sido considerados mais impactantes.

Diante disso, a proposta deste trabalho de iniciação científica consiste em investigar técnicas para entender quais os tópicos comentados e quais são os sentimentos dos usuários do Twitter em relação a pandemia da COVID-19. Nesse sentido, algumas Perguntas de Pesquisa (PP) foram elaboradas para guiar o escopo do trabalho:

- PP1: Quais são os melhores modelos/ferramentas para realizar uma análise de sentimento no contexto da pandemia da COVID-19?

- PP2: Quais são os melhores algoritmos para modelagem de tópicos no mesmo contexto citado no PP1?

- PP3: Como os tópicos encontrados na modelagem se relacionam com a classificação de sentimentos?

Com o objetivo de responder as perguntas de pesquisa levantadas, neste trabalho são relatados os experimentos para a avaliação de ferramentas de classificação de sentimento e modelagem de tópicos aplicados ao tema.

\section{TRABALHOS RELACIONADOS}

Xiang e Zhou [12] propõem diversas abordagens para melhorar a análise de sentimento em dados do Twitter. O trabalho propõe um modelo semelhante ao estudo atual, com a combinação de sentimento com tópicos específicos, entretanto, em uma estrutura de treinamento semi-supervisionada. Para a modelagem de tópicos é utilizado o Latent Dirichlet Allocation (LDA). Em seus resultados, mostram superar o sistema estado-da-arte no SemEval-2013.

Jeong et al. [6] realizam uma abordagem de mineração de mídia social para a exploração de oportunidades de produtos. A proposta é semelhante ao estudo deste trabalho, é baseada na modelagem de tópicos e análise de sentimento em tais mídias sociais. Consiste na identificação dos níveis de oportunidade e as direções de melhoria dos tópicos do produto. O artigo contribui para a descoberta sistemática de oportunidades de produtos nas mídias sociais.

Cer et al. [1] propõem a análise de conteúdo através de sentimentos baseado em séries temporais, especificamente na base diária e modelagem de tópicos, também com base LDA, para avaliar o sentimento do tópico bem como o modelo dos tópicos discutidos. $\mathrm{O}$ trabalho contribuiu com empresas e indivíduos no mapeamento da opinião pública em relação a determinado tópico, analisando o sentimento do texto e criando um modelo de tópico. 
Kaur et al. [7] trouxeram a proposta de analisar os sentimentos em relação à doença coronavírus (COVID-19). De forma semelhante ao presente trabalho, a partir dos tweets de streaming relacionados a esse tema, técnicas e ferramentas de aprendizado de máquina foram aplicadas para a classificação desses compartihamentos como positivos, negativos ou neutros. Os experimentos envolveram a programação em Python usando, além da API do Twitter, a biblioteca NLTK $^{1}$ para o pré-processamento antes da análise final usando a ferramenta Textblob ${ }^{2}$.

\section{METODOLOGIA}

A metodologia utilizada neste trabalho consiste em 4 etapas de desenvolvimento: 1) coleta do dataset; 2) preparação; 3) análise de sentimentos; 4) modelagem de tópicos.

\subsection{Coleta do Dataset}

Nesta fase selecionou-se um dataset de mensagens do Twitter relacionados à pandemidia do COVID-19. O dataset escolhido é chamado "Coronavirus tweets NLP - Text Classification"3 e possui todos os tweets no idioma inglês. Ele é divido em dois conjuntos, um de treino e outro para teste. O conjunto de treino possui 41.157 exemplos, enquanto o conjunto de teste possui 3.798 exemplos. $\mathrm{O}$ dataset está anotado com cinco classes de sentimentos: Extremely Negative, Negative, Neutral, Positive, e Extremely Positive.

\subsection{Preparação}

A fim de obter uma análise adequada sobre os dados obtidos, é realizada uma etapa de preparação dos itens com o intuito de remover inconsistências do grupo de informações. Para isso, a ferramenta Natural Language Toolkit (NLTK) foi utilizada seguindo um padrão que consiste de:

(1) Transformação para caixa baixa;

(2) Remoção de stopwords;

(3) Remoção de caracteres numéricos;

(4) Remoção de pontuações ou caracteres especiais;

Adicionalmente, as cinco classes de sentimentos originais do dataset foram simplificadas para três. Os exemplos da classe Extremely Negative foram re-anotados para a classe Negative, enquanto os exemplos da classe Extremely Positive foram re-anotados para a classe Positive.

\subsection{Análise de sentimentos}

Para a realização da análise de sentimentos, foi desenvolvido um modelo para classificação de sentenças de texto em 3 classes de sentimentos (Negative, Neutral, e Positive). Conforme ilustrado na Figura 1, o modelo é composto de dois módulos: o Universal Sentence Encoder e o Classificador de Sentimento.

O módulo Universal Sentence Encoder [3] consiste da arquitetura Transformer [11] pré-treinada em uma base universal. Dado uma sentença de texto, o Universal Sentence Encoder converte a sentença para um vetor de features com 512 dimensões (embedding), o qual possui informação semântica de alto nível.

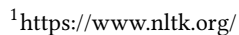

${ }^{2}$ https://textblob.readthedocs.io/en/dev/

${ }^{3}$ https://www.kaggle.com/datatattle/covid-19-nlp-text-classification
}

O módulo Classificador de Sentimento consiste de uma rede neural do tipo MLP [4], e está estruturada da seginte maneira: 1) camada de entrada com 512 neurônios; 2) primeira camada escondida com 1024 neurônios; 3) segunda camada escondida com 512 neurônios; 4) camada de saída com 3 neurônios, um para cada classe. Vale ressaltar que apenas o Classificador de Sentimento é treinado; o Universal Sentence Encoder permanece com os pesos congelados e é usado apenas para conversão das sentenças de texto em embeddings.

Adicionalmente, também foram utilizadas outras ferramentas para a análise de sentimentos, como o Polyglot e TextBlob. A motivação para a criação e treinamento de um modelo próprio de classificação surgiu por causa do fraco desempenho dessas ferramentas no dataset escolhido no âmbito do projeto.

\subsection{Modelagem de tópicos}

Neste projeto foram utilizados três algoritmos de modelagem, o Non-Negative Matrix Factorization (NMF), o Latent Dirichlet Allocation (LDA) e o Latent Semantic Analysis (LSA), todos baseados em modelos estatísticos próprios. O LDA é um modelo estatístico que gera coleções de dados discretos a partir de informações semelhantes [2]. O LSA é caracterizado como uma técnica de análise da relação de termos em conjuntos de documentos [5]. E por fim, o NMF é considerado um grupo de algoritmos em análise multivariada para dados dimensionais elevados, pois extrai automaticamente recursos significativos de um conjunto de vetores de dados não negativos [8].

\section{EXPERIMENTO}

Foi realizado um experimento para atestar a qualidade do modelo de classificação de sentimentos e seleção do algoritmo de modelagem de tópicos. O código fonte, os pesos do treinamento, e o dataset utilizado na experimentação se encontram disponíveis no repositório deste projeto no Github. ${ }^{4}$ As próximas subseções detalham, respectivamente, as configurações do experimento, e os resultados obtidos.

\subsection{Configuração}

Para realização do experimento, o conjunto de teste do dataset foi divido pela metade, resultando em 1.899 exemplos para o conjunto de validação, com o restante permanecendo no conjunto de teste.

Na rede MLP usada para análise de sentimentos, foram usados os seguintes hiper-parâmetros: ativação ReLU nas três primeiras camadas, ativação linear na última camada, inicialização Xavier, Optimizador Adam com taxa de aprendizagem de 0.001, e entropia cruzada com softmax como função de perda.

\subsection{Resultados}

4.2.1 Análise de sentimentos. Conforme descrito na Tabela 1, o melhor resultado foi obtido pelo modelo "Transformer Encoder + MLP", que obteve um F1-Score de 68,87\% no conjunto de validação, respondendo assim à PP1. Em seguida, a ferramenta Polyglot ficou em segundo lugar, produzindo um F1-Score de 59.44\%. Por fim, a ferramenta TextBlob ficou no terceiro lugar, produzindo um F1Score de $53.07 \%$.

\footnotetext{
$\overline{{ }^{4} \text { https://github.com/TeleMidia/Text-Sentiment-Analysis }}$
} 
Texto do tweet

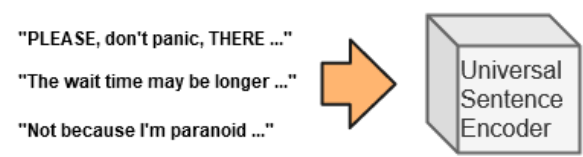

Embedding

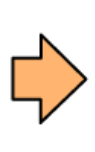

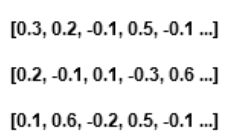

Classificação

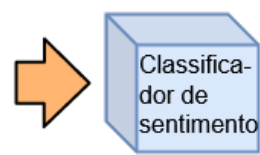

Positive

Positive

Negative

Figure 1: Modelo para classificação de sentimentos

\begin{tabular}{|c|c|c|c|}
\hline Modelo & F1-Score & Precisão & Recall \\
\hline Transfor. Encoder + MLP & $68,87 \%$ & $68,89 \%$ & $68,85 \%$ \\
\hline Polyglot & $59.44 \%$ & $57.47 \%$ & $66.93 \%$ \\
\hline TextBlob & $53.07 \%$ & $55.19 \%$ & $53.05 \%$ \\
\hline
\end{tabular}

Table 1: Comparação dos resultados no conjunto de validação.
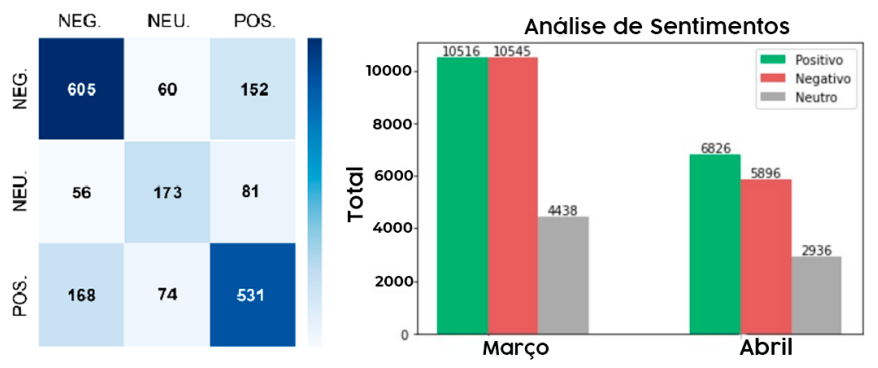

Figure 2: (Esquerda) Matriz de confusão do melhor modelo no conjunto de teste. (Direita) Distribuição em meses de sentimentos classificados nos tweets

A Tabela 2 mostra o resultado do melhor modelo no conjunto de teste. O resultado produzido foi similar ao obtido no conjunto de validação. Mais detalhadamente, a Figura 2 (esquerda) exibe a matriz de confusão entre as 3 classes no conjunto de teste. Notase que o modelo consegue diferenciar bem os tweets negativos e positivos, mas ainda há muitos erros na classe neutro.

\begin{tabular}{|c|c|c|c|}
\hline Modelo & F1-Score & Precisão & Recall \\
\hline Transfor. Encoder + MLP & $68.79 \%$ & $68.86 \%$ & $68.96 \%$ \\
\hline
\end{tabular}

Table 2: Resultado do melhor modelo no conjunto de teste.

Finalizada a análise com o modelo "Transformer Encoder + MLP", foram obtidos 17.342 casos positivos, 16.441 casos negativos e 7.374 casos neutros. Nota-se que a parte predominante dos tweets são de casos positivos, com $42,13 \%$ da distribuição geral. Em seguida, os tweets negativos apresentam um total de aproximadamente $40 \%$, seguidos pelos neutros com apenas $17.91 \%$. A análise foi realizada nos meses de março e abril de 2020. a figura 2 (direita) mostra os dados de forma gráfica.

4.2.2 Modelagem de Tópicos. No experimento da modelagem de tópicos, três algoritmos foram testados: Non-Negative Matrix Factorization (NMF); Latent Dirichlet Allocation (LDA); Latent Semantic Analysis (LSA). Os algoritmos foram avaliados de forma qualitativa pelos membros do projeto a partir da identidicação dos tópicos gerados por cada algoritmo. O agrupamento de palavras gerado pelo NMF possuía maior relação entre os termos e maior coerência relacionada ao tema de pesquisa, respondendo assim à PP2. Com isso, o Term Frequency Inverse Document Frequency (TF-IDF), em conjunto com NMF foram aderidos ao trabalho com o objetivo de classificar as palavras em ordem de importância no conjunto de tweets, demonstrado de forma semelhante em [10].

A Tabela 3 mostra os tópicos que foram encontrados. Percebe-se que há relação na ocorrência das palavras encontradas e a discussão relacionada a pandemia do COVID-19. Isso mostra a importância da modelagem relacionado à classificação de sentimentos aplicado aos assuntos que são comentados ao redor do mundo. Os tópicos variam em vários âmbitos da problemática, como economia, medicina, prevenção e demais assuntos que estiveram em alta durante a pandemia.

\begin{tabular}{|l|l|}
\hline Tópico & Palavras \\
\hline Shopping & covid shopping online virus get home \\
\hline Fuel & prices covid oil low pandemic gas \\
\hline Cleaning & coronavirus prices toiletpaper hand pandemic trump \\
\hline Supermarket & supermarket panic shelves stop empty buy \\
\hline Food & food panic demand stock supply due \\
\hline Health & crisis covid workers health essential staff \\
\hline Grocery & store grocery workers retail food go \\
\hline Prevention & masks us health risk people social \\
\hline Scams & consumer crisis pandemic online new scams \\
\hline Spread & covid store pandemic help one spread \\
\hline Quarantine & coronavirus corona paper toilet quarantine pandemic \\
\hline Home & home stay safe keep covid everyone \\
\hline Prices & prices people price us get like \\
\hline Lockdown & covid coronavirus toiletpaper pandemic lockdown \\
\hline Consume & consumer impact pandemic new amp us \\
\hline
\end{tabular}

Table 3: Modelagem de tópicos encontrados.

A Tabela 4 mostra a relação entre as 3 classes de sentimentos e os tópicos encontrados. Percebe-se a presença de 9 tópicos negativos distintos. Dentre eles, alguns assuntos tiveram repercussões exclusivamente negativas. O tópico Fuel que se retrata da queda no preço do petróleo, e consequentemente dos combustíveis; em Cleaning com o aumento do preço de materiais de limpeza e as medidas de saúde do governo Trump; em Food retratando o pânico causado em algumas pessoas e gerando a necessidade de alguns em estocar alimentos, gerando falta nos supermercados; e por fim, Scams com o aumento de golpes pela internet, uma vez que com a quarentena, o número de compras online subiu bastante.

A respeito dos tópicos positivos, há o Spread, que retrata a propagação do vírus e como é importante a conscientização das pessoas 


\begin{tabular}{|c|c|c|c|}
\hline Tópico & Positivo & Negativo & Neutro \\
\hline Shopping & $\mathbf{X}$ & $\mathbf{X}$ & $\mathbf{X}$ \\
\hline Fuel & & $\mathbf{X}$ & $\mathbf{X}$ \\
\hline Cleaning & & $\mathbf{X}$ & $\mathbf{X}$ \\
\hline Supermarket & $\mathbf{X}$ & $\mathbf{X}$ & $\mathbf{X}$ \\
\hline Food & & $\mathbf{X}$ & $\mathbf{X}$ \\
\hline Health & $\mathbf{X}$ & $\mathbf{X}$ & \\
\hline Grocery & $\mathbf{X}$ & $\mathbf{X}$ & $\mathbf{X}$ \\
\hline Prevention & $\mathbf{X}$ & $\mathbf{X}$ & \\
\hline Scams & & $\mathbf{X}$ & \\
\hline Spread & $\mathbf{X}$ & & \\
\hline Quarantine & $\mathbf{X}$ & & $\mathbf{X}$ \\
\hline Home & $\mathbf{X}$ & & $\mathbf{X}$ \\
\hline Prices & $\mathbf{X}$ & & \\
\hline Lockdown & & & $\mathbf{X}$ \\
\hline Consume & & & $\mathbf{X}$ \\
\hline
\end{tabular}

Table 4: Correlação de tópicos encontrados por sentimento nos tweets.

para a retenção da doença; em Quarantine e Home mostrando a conscientização de partes da população a respeito do isolamento social; e com Prices comentando a alteração de preços em vários setores da economia.

Vale ressaltar a existência de tópicos que foram modelados tanto com sentimentos negativos, quanto positivos, como Shopping, Supermarket, Health, Grocery e Prevention. Diante disto, uma análise de maneira separada foi realizada. No tópico Shopping surgiram comentários negativos sobre fazer compras na época da pandemia e seus riscos por conta do vírus, por outro lado, comentários positivos apareceram sobre permanecer em casa, fazer compras pela internet e pedidos de delivery; em Supermarket, algumas pessoas comentaram negativamente a respeito do medo em vários lugares do mundo com a falta de comida nos supermercados, das grandes filas, e do aumento em alguns produtos, por outro lado, vários comentários positivos a respeito do trabalho das equipes nos supermercados, dos cuidados e serviços prestados; em Health os comentários negativos se tratavam basicamente ao perigo que os profissionais de saúde estavam correndo todos os dias na linha de frente contra o coronavírus, e com comentários positivos a respeito justamente desses profissionais, em agradecimento pela luta e proteção contra a COVID-19; em Grocery os comentários negativos eram sobre as vendas nas mercearias e como estavam sendo realizadas, entretanto, os positivos retravam justamente como esse meio de venda se tornou a renda fixa de várias pessoas durante a época de pandemia; e por fim, Prevention que retratava negativamente comentários a respeito do risco ao não tomar as iniciativas preventivas, e o assunto foi abordado positivamente retratando os benefícios dessas iniciativas, como o uso de máscaras. luvas e lavar as mãos com sabão. Diante do exposto, temos então a resposta para a PP3.

\section{CONSIDERAÇÕES FINAIS}

Este artigo apresentou um estudo de caso sobre o relacionamento da modelagem de tópicos com a análise de sentimentos no contexto da pandemia do COVID-19. Para isso, um dataset de mensagens do Twitter, chamado "Coronavirus tweets NLP - Text Classification" foi utilizado para treinar um classificador de sentimentos. O conjunto de teste foi dividido em três partes, um para cada classe de sentimento predito pelo classificador. Em seguida, algoritmo NMF foi utilizado para gerar a lista de tópicos para cada conjunto de sentimento. Por fim, foi realizada uma análise dos tópicos encontrados em cada conjunto de sentimentos.

Após a aplicação do classificador de sentimentos, percebeu-se que os sentimentos das classes positiva e negativa ficaram em quantidade semelhantes, apesar do sentimento positivo ter uma quantidade um pouco maior. Em conjunto com a modelagem de tópicos, tal análise torna poissível um maior entendimento das opiniões e assuntos úteis que fazem parte do contexto da pandemia da COVID19 , trazendo uma ferramenta que pode servir para compreensão dos acontecimentos discutidos no cotidiano.

Como trabalhos futuros, pode-se citar a utilização de outras técnicas de classificação de sentimentos a fim de buscar resultados melhores do que o "Transformer Encoder + MLP". Adicionalmente, pretende-se aumentar a base de tweets sobre a pandemia da COVID19, com capturas de mais meses do ano de 2020. Com isso, será realizada a análise dos tópicos em períodos de tempo diferentes para entender como os sentimentos e tópicos abordados pelos usuários mudaram no decorrer do tempo.

\section{REFERENCES}

[1] Andry Alamsyah, Wirawan Rizkika, Ditya Dwi Adhi Nugroho, Farhan Renaldi, and Siti Saadah. 2018. Dynamic large scale data on Twitter using sentiment analysis and topic modeling. In 2018 6th International Conference on Information and Communication Technology (ICoICT). IEEE, 254-258.

[2] David M Blei, Andrew Y Ng, and Michael I Jordan. 2003. Latent dirichlet allocation. Journal of machine Learning research 3, Jan (2003), 993-1022.

[3] Daniel Cer, Yinfei Yang, Sheng-yi Kong, Nan Hua, Nicole Limtiaco, Rhomni St John, Noah Constant, Mario Guajardo-Cespedes, Steve Yuan, Chris Tar, et al. 2018. Universal sentence encoder. arXiv preprint arXiv:1803.11175 (2018).

[4] Simon S Haykin et al. 2009. Neural networks and learning machines/Simon Haykin.

[5] Thomas Hofmann. 2013. Probabilistic latent semantic analysis. arXiv preprint arXiv:1301.6705 (2013), 289-296.

[6] Byeongki Jeong, Janghyeok Yoon, and Jae-Min Lee. 2019. Social media mining for product planning: A product opportunity mining approach based on topic modeling and sentiment analysis. International fournal of Information Management 48 (2019), 280-290.

[7] Chhinder Kaur and Anand Sharma. 2020. Twitter Sentiment Analysis on Coronavirus using Textblob. EasyChair Preprint no. 2974.

[8] Daniel D Lee and H Sebastian Seung. 2001. Algorithms for non-negative matrix factorization. In Advances in neural information processing systems. 556-562.

[9] Hussin A Rothan and Siddappa N Byrareddy. 2020. The epidemiology and pathogenesis of coronavirus disease (COVID-19) outbreak. fournal of autoimmunity (2020), 102433.

[10] Joni Salminen, Hind Almerekhi, Milica Milenkovic, Soon-gyo Jung, Jisun An, Haewoon Kwak, and Bernard J Jansen. 2018. Anatomy of Online Hate: Developing a Taxonomy and Machine Learning Models for Identifying and Classifying Hate in Online News Media.. In ICWSM. 330-339.

[11] Ashish Vaswani, Noam Shazeer, Niki Parmar, Jakob Uszkoreit, Llion Jones, Aidan N Gomez, Łukasz Kaiser, and Illia Polosukhin. 2017. Attention is all you need. In Advances in neural information processing systems. 5998-6008.

[12] Bing Xiang and Liang Zhou. 2014. Improving twitter sentiment analysis with topic-based mixture modeling and semi-supervised training. In Proceedings of the 52nd Annual Meeting of the Association for Computational Linguistics (Volume 2: Short Papers). 434-439. 